\title{
Anti-malarial Drugs as Potential Inhibitors of Leishmania Glycolytic Enzymes: Development of New Anti-leishmanial Agents
}

\author{
Bashir A. Yousef ${ }^{1}$, Tanzeel H. Elwaseela², Tagwa A. Ali², Fatima E. Mohammed², \\ Wala O. Mohammed ${ }^{2}$, Majdi Alobaid ${ }^{2}$, Amina I. Dirar ${ }^{3}$ \\ ${ }^{1}$ Department of Pharmacology, Faculty of Pharmacy, University of Khartoum, \\ Al-Qasr Ave, Khartoum 11111, Sudan \\ ${ }^{2}$ Department of Pharmacology, Faculty of Pharmacy, National Ribat University, \\ Bori Street, Khartoum 11111, Sudan \\ ${ }^{3}$ Medicinal, Aromatic Plants and Traditional Medicine Research Institute, \\ National Centre for Research, Sudan, Almek-Nimir Street, Khartoum 11111, Sudan
}

\begin{abstract}
Leishmaniasis is one of the most important endemic diseases in Sudan. The glycolytic pathway is one of the essential pathways in the survival and pathogenicity of the leishmania parasite. This study aimed to evaluate the antileishmanial activities of three antimalarial drugs through targeting the glycolytic pathway of the parasite. Anti-leishmanial activities of artesunate, quinine and mefloquine were evaluated using an in vitro anti-promastigote assay. Then, in silico molecular docking was conducted using Autodock 4.0 software to study the molecular interactions of antimalarial drugs to different key glycolytic enzymes. The results of the current study, Artesunate, quinine, and mefloquine showed effective inhibitory activities against L. donovani with IC50 values of 58.85, 40.24, and $20.06 \mu \mathrm{g} / \mathrm{ml}$, respectively. Molecular docking analysis revealed interesting interactions between different antimalarial drugs and various glycolytic enzymes (Glucose-6-phosphate isomerase, Triosephosphate isomerase, Glycerol-3-phosphate dehydrogenase, Glyceraldehyde-3-phosphate dehydrogenase and Pyruvate kinase). Moreover, these drugs interact with different amino acid residues of the proteins with satisfactory binding energies, particularly with artesunate. According to binding energies, Glycerol-3-phosphate dehydrogenase was represented the most potential target for three tested drugs. Collectively, our results showed promising antileishmanial activities of different antimalarial that may mediated through inhibition of glycolysis process in leishmania donovani promastigote.
\end{abstract}

Keywords: Anti-malarial drugs, Anti-leishmanial activity, Glycolytic pathway, Molecular docking, Glycerol-3-phosphate dehydrogenase.

\section{Introduction}

A leishmaniasis is a group of parasitic diseases caused by Leishmania species, which is transmitted by the bite of a Lutzomyia or Phlebotomus sand fly. ${ }^{1,2}$ Leishmaniasis mainly occurs in most tropical and subtropical countries. ${ }^{3,4}$ However, more than $90 \%$ of cases occur in Afghanistan, Algeria, Bangladesh, Bolivia, Brazil, Columbia, Ethiopia, India, Iran, Peru, South Sudan, Sudan and Syria. ${ }^{3}$

Corresponding author: Bashir A. Yousef. Department of Pharmacology, Faculty of Pharmacy, University of Khartoum. Email: bashiralsiddiq@gmail.com

Received: 8 September 2020. Revised: 20 November 2020 . Published: 3 December 2020 
One of the most important endemic diseases in Sudan is visceral leishmaniasis (VL). Sudan represents one of the main VL foci globally, as $90 \%$ of VL incidence appears in 5 countries (India, Bangladesh, Brazil, Nepal, and Sudan). ${ }^{3,5,6} \mathrm{VL}$ was spread over a wide belt along the Sudanese-Ethiopian border from the Atbara River in the northeast and extending west across the White Nile to the Sobat River in South Sudan. It was focused in the Kapoeta area, the Nuba Mountains and Nassir, and Malakal and scattered areas in the Darfur region. ${ }^{5,7,8}$

The glycolytic pathway is one of the essential pathways in the survival and pathogenicity of the Leishmania parasite. ${ }^{9}$ Due to the lack of the functional Krebs cycle, the parasite utilizes the host carbons as a source of energy through the glycolytic pathway, representing the only source for Adenosine triphosphate (ATP) generation inside the parasite. ${ }^{10,11}$ Therefore, inhibition of the glycolytic pathway by various agents could be considered as an excellent strategy to target leishmaniasis, as the glycolysis is the only source of energy. ${ }^{12}$

The leishmania glycolytic pathway consists of multiple enzymes that play an essential role in the cascade of the glycolysis process, such as pyruvate kinase ${ }^{13}$, glucose6-phosphate isomerase ${ }^{14}$, UDP-glucose pyrophosphorylase ${ }^{15}$, glyceraldehyde-3phosphate dehydrogenase ${ }^{16}$, glycerol-3phosphate dehydrogenase ${ }^{17}$, triosephosphate isomerase ${ }^{18}$, thiol-dependent reductase $1^{19}$, and phosphomannomutase..$^{20}$

Most anti-leishmanial drugs are toxic and less effective, and most of these medications are used parenterally for prolonged periods, especially for visceral leishmaniasis; also, it has several limitations drug-resistance, length of treatment, and cost lines. ${ }^{21,22}$ So, there is an urgent need to search for new antileishmanial compounds of advantage that can outweigh the problem of the available anti-leishmanial drugs. In Sudan, malaria is co-endemic with visceral leishmaniasis, and co-infections with both diseases are common. ${ }^{23}$ Previous clinical observation from Ecuador showed that significant efficacy of anti-malarial drugs, mefloquine and artesunate against leishmania. ${ }^{24}$ In this study, the in vitro and in silico evaluation of the efficacy of different antimalarial drugs, including artesunate, quinine and mefloquine for their potential antileishmanial activity was assessed.

\section{Methods}

Parasite culture

L. donovani promastigotes used in this study were kindly provided by the Department of Microbiology, Medicinal, Aromatic Plants and Traditional Medicine Research Institute (MAPTRI), Khartoum, Sudan. Promastigotes were cultured at $26^{\circ} \mathrm{C}$ in culture flasks containing RPMI 1640 medium (Sigma, St. Louis, MO) and supplemented with 5\% fetal bovine serum (FBS), $2 \mathrm{mM}$ glutamine, 100 units/ml penicillin, and 100 $\mathrm{mg} / \mathrm{ml}$ streptomycin (Sigma, St. Louis, MO). Promastigotes were harvested after four or five days of incubation, and the growth of it was observed daily by using an inverted microscope (CK40; Olympus, Tokyo, Japan).

\section{In vitro Evaluation Against Leishmania Donovani Promastigotes}

The in vitro anti-promastigote assay was performed as previously described with trivial modifications 25. Briefly, the harvested promastigotes of $L$. donovania were suspended in a concentration of $(2 \times 106$ parasites $/ \mathrm{ml}$ ) in culture medium and then incubated in 96-well microtiter plate at 26 ${ }^{\circ} \mathrm{C}$ for $72 \mathrm{hr}$ in fresh medium, in the absence and the presence of antimalarial drugs (artesunate, quinine, and mefloquine) at several concentrations. $40 \mu \mathrm{l}$ of the maximum 
concentrations of the drugs solutions artesunate $(500 \mu \mathrm{g} / \mathrm{ml})$, quinine $(600 \mu \mathrm{g} / \mathrm{ml})$, and mefloquine $(100 \mu \mathrm{g} / \mathrm{ml})$ were added in the first column wells (C-1). $20 \mu$ l of complete RPMI 1640 medium (HyClone China Ltd., China) was added to the other wells (C-2 and C-6). Serial dilutions of the drugs were performed by taking $20 \mu \mathrm{l}$ from the previous wells and added to next wells. Then, $80 \mu \mathrm{l}$ of culture medium that contained the parasite was added to each well. Pentamidine was used as positive control whereas untreated cells were used as a negative control (culture medium plus parasites). For checking the activity, trypan blue were mixed with the samples, and then counted using by using a hemocytometer with a $20 \times$ objective under standard light microscopy. The results were represented as the mortality rate $\%$ and the mean inhibitory concentration $\left(\mathrm{IC}_{50}\right)$ after 72 $\mathrm{h}$ of incubation period.

\section{In silico Molecular Docking Study}

The 3-D structure of five enzymes involved in glycolysis; glucose-6-phosphate isomerase (PDB ID: 1T10) ${ }^{14}$, triosephosphate isomerase (PDB ID: 2Y63) 18, glycerol-3-phosphate dehydrogenase (PDB ID: 1M67) 17, glyceraldehyde-3-phosphate dehydrogenase (PDB ID: 1I33) 16, and pyruvate kinase (PDB ID: 3PP7) 13, were retrieved from the RCSB Protein Data Bank in .pdb format at models quality of $2.35 \AA, 1.97 \AA, 2.50 \AA$, $3.00 \AA$, and $2.35 \AA$ resolution, respectively. The surface features and functional regions of the proteins were determined using the Computed Atlas for Surface Topography of Proteins (CASTp 3.0) web server http://sts. bioe.uic.edu/castp/. ${ }^{26}$

Three anti-malarial compounds; artesunate, quinine, and mefloquine were selected as potential candidates for drug repositioning for leishmaniasis. The anti-malarial compounds and the positive control pentamidine (Figure
1) were docked into the selected enzymes of glycolytic pathway. Molecular docking was carried out using Autodock 4.0 software ${ }^{27}$, based on Lamarckian Genetic Algorithm. ${ }^{28}$ The protein structure was prepared by removal of water molecules and hetero groups. Then, we were added the polar hydrogen atoms to the protein targets, and computed the Kollman united atomic charges. The default docking algorithms were set following standard docking protocol. Ten independent docking runs were carried out for each ligand and results were retrieved as binding energies. Poses that showed lowest binding energies were visualized using BIOVIA Discovery Studio Visualizer (DVS) software. ${ }^{29}$ Target proteins were re-docked with their cocrystallized ligand for validation of docking protocol.

\section{Statistical analysis}

All tests were repeated three times. The results were expressed as the mean $\pm \mathrm{SD}$. A two tailed Student's test was used to evaluate the significance of the results. Data were analyzed using GraphPad Prism 6 (GraphPad Software, Inc., San Diego, CA, USA) and $\mathrm{P}$ value $<0.05$ was considered statistically significant.

\section{Results and discussion}

A reduction of the number of viable promastigotes was noticed after incubation for $72 \mathrm{~h}$ with different concentrations of various antimalarial drugs, and the reduction was significantly increased with the increasement of concentrations of these drugs (Figure 2). The $\mathrm{IC}_{50}$ value of the positive control anti-leishmania drug, pentamidine was $1.3 \pm 0.37 \mu \mathrm{g} / \mathrm{mL}$, while the other tested anti-malarial compounds; quinine, artesunate and mefloquine showed less $\mathrm{IC}_{50}$ values of $40.24 \pm 8.24 \mu \mathrm{g} / \mathrm{mL}, \quad 58.85 \pm 9.73 \mu \mathrm{g} / \mathrm{mL}$, $20.06 \pm 4.1 \mu \mathrm{g} / \mathrm{mL}$, respectively. 
Table 1. Protein-ligand Binding Free Energy (kcal/mol)

\begin{tabular}{lcccc}
\hline \multicolumn{1}{c}{ Protein } & \multicolumn{4}{c}{ Binding Energy (kcal/mol) } \\
\cline { 2 - 5 } & Pentamidine* & Artesunate & Quinine & Mefloquine \\
\hline $\begin{array}{l}\text { Glucose-6-phosphate isomerase } \\
\text { (PDB ID: 1T10) }\end{array}$ & -5.32 & -6.46 & -6.14 & -6.51 \\
\hline $\begin{array}{l}\text { Triosephosphate isomerase } \\
\text { (PDB ID: 2Y63) }\end{array}$ & -5.41 & -7.78 & -6.12 & -6.11 \\
\hline $\begin{array}{l}\text { Glycerol-3-phosphate dehydrogenase } \\
\text { (PDB ID: 1M67) }\end{array}$ & -7.69 & -9.31 & -7.43 & -6.70 \\
\hline $\begin{array}{l}\text { Glyceraldehyde-3-phosphate dehydro- } \\
\text { genase (PDB ID: 1I33) }\end{array}$ & -6.85 & -7.94 & -6.72 & -6.91 \\
\hline $\begin{array}{l}\text { Pyruvate kinase } \\
\text { (PDB ID: 3PP7) }\end{array}$ & -6.22 & -8.74 & -6.07 & -5.37 \\
\hline
\end{tabular}

* Positive Control

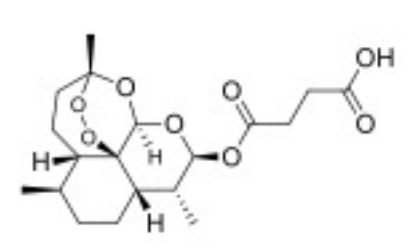

Artesunate

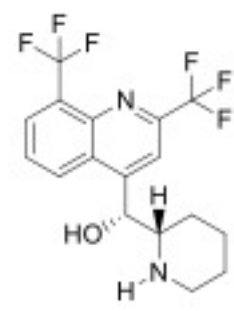

Mefloquine

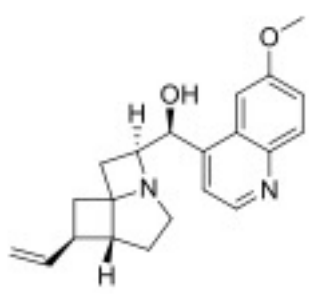

Quinine<smiles>N=C(N)c1ccc(OCCCCCOc2ccc(C(=N)N)cc2)cc1</smiles>

Pentamidine

Figure 1. Chemical Structures of The Anti-malarial Compounds and The Positive Control Pentamidine

The results in hand suggest fair antileishmanial activities of these compounds as compared to the positive control pentamidine. The potential anti-leishmanial activity for quinine, artesunate and mefloquine had been reported in different studies. Herein, a previous study had reported the antileishmanial activity for quinine against $L$. donovani. ${ }^{30}$. Also, a recent finding reported the effective anti-leishmanial activity of quinine formulated as quinine sulphate microparticles ${ }^{31}$, and this could plausibly explain the poor activity for quinine in our finding. Similarly, mefloquine was also reported to be effective as anti-leishmanial agent and exerts a noticeable toxic effect against promastigotes in axenic cultures among other tested antimalarial drugs. ${ }^{32}$ Artesunate reported a poor anti-leishmanial activity as compared to other tested drugs. ${ }^{33,34}$ It was reported previously the divergence of artesunate activity as anti-leishmanial agent and pertained to the difference in genotypes between Leishmania species. ${ }^{35}$ 
A

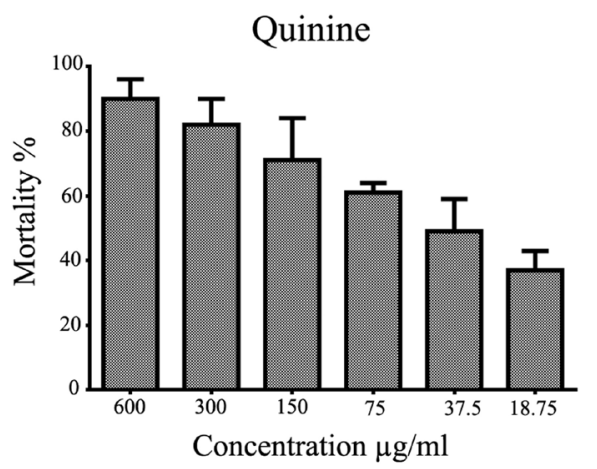

B

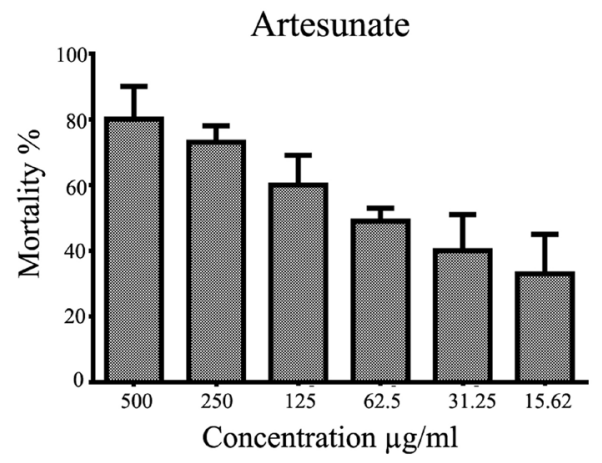

$\mathrm{C}$

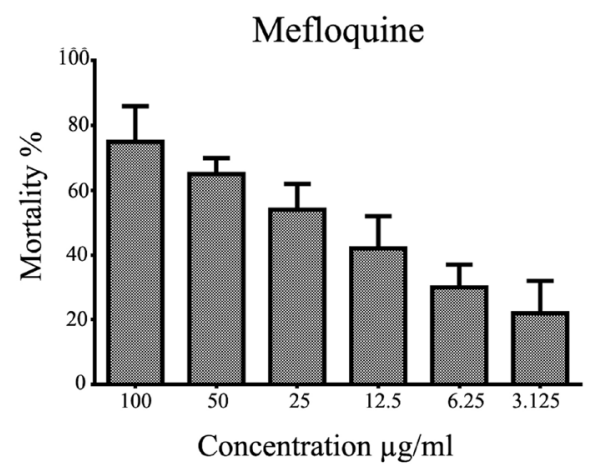

Figure 2. Antileishmanial activity of antimalarial drugs against Leishmania donovani promastigotes

Briefly, harvested promastigotes of L. donovania were cultured in 96-well microtitre plates and treated with different antimalarial drugs (artesunate, quinine and mefloquine) then mortality rate $\%$ was measured after $72 \mathrm{~h}$ of incubation period.

Molecular docking has been used efficiently for drug repurposing predictions. ${ }^{36}$ Thus, we have attempted to study the possible interactions that would occur between the compounds and enzymes involve in glycolysis pathway. Table 1 and Figure 3-6 showed the interactions between the antimalarial drugs and the positive control pentamidine with several key proteins in the parasite glycolysis pathway. Glycolysis is a vital and sole metabolic pathway for ATP supply in the parasite. ${ }^{14}$. The initial enzymes involved in glycolysis are sequestered in the glycosome, a specialized organelle, and thus inhibition of these enzymes block the growth of the parasite. ${ }^{37}$. So far, the molecular 


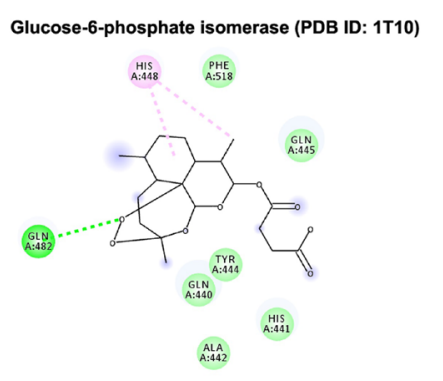

Glyceraldehyde-3-phosphate dehydrogenase (PDB ID: 1133)

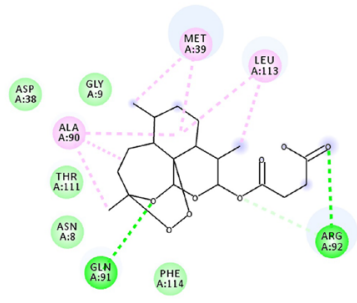

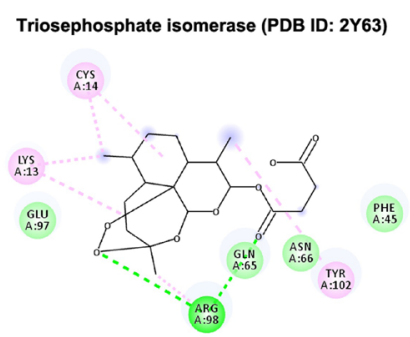

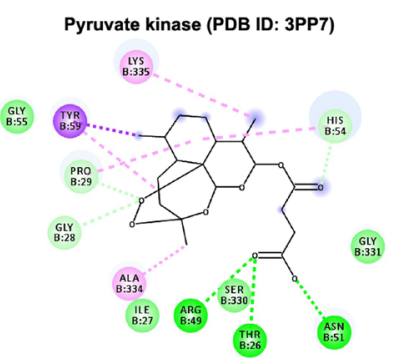

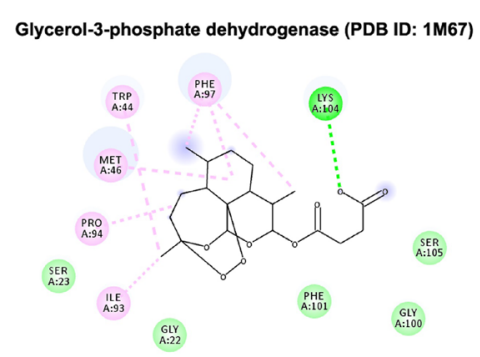

Interactions
$\square$ van der Waals
$\square$ Conventional Hydrogen Bond
$\square$ Carbon Hydrogen Bond
$\square$ Pi-Sigma
$\square$ Alkyl
$\square$ Pi-Alkyl

Figure 3. Artesunate Interaction with Different Amino Acid Residues of Glycolytic Enzymes;

Glucose-6-phosphate isomerase (PDB ID: 1T10), Triosephosphate isomerase (PDB ID: 2Y63), Glycerol-3-phosphate dehydrogenase (PDB ID: 1M67), Glyceraldehyde3-phosphate dehydrogenase (PDB ID: 1I33), pyruvate kinase (PDB ID: 3PP7). Different kinds of interaction between amino acid residues and artesunate were demonstrated inside the figure. Interactions were visualized by BIOVIA DVS.

docking studies on anti-malarial drugs against leishmaniasis have not been investigated in details. This had driven us to assess the possible molecular binding interaction of these compounds with enzymes involved in glycolysis. As for the enzyme glucose6-phosphate isomerase (PGI), the positive control pentamidine have shown hydrogen bonding with the catalytic residues HIS-441 and ALA-442 and a relatively good binding affinity (-5.32 Kcal/mol). While docking results of the other compounds have showed merit binding interaction with a 10-residue loop (of amino acids GLY-433 to ALA-442) in which the catalytic HIS-441 is located (Figure 3,4 and 5) $)^{14}$.

All compounds have not interacted with MET-337 located in the $\alpha$-helical segment connecting the $\mathrm{C}$-terminal side of the small domain to the LH-c of the large domain of the enzyme. Moreover, the compounds have not shown biochemical interactions with both residues HIS-388 and GLU-357 which participates in the substrate ring opening step and isomerization step, respectively. ${ }^{14}$ From table 1, it's clear that all compounds showed lowest binding energy in comparison to pentamidine $(-6.51,-6.46$, and $-6.14 \mathrm{Kcal} /$ mol for mefloquine, artesunate and quinine, respectively).

As triosephosphate isomerase (TIM), docking result for pentamidine showed three conventional hydrogen bonding with CYS14, ASN-15 and GLN-65 and other favorable interactions with the residues HIS-95 and GLU-97. While the docked compounds were located around the four catalytic residues ASN-11, LYS-13, HIS-95, and GLU-167 at the dimer interface. However, the compounds have not shown biochemical interactions with 


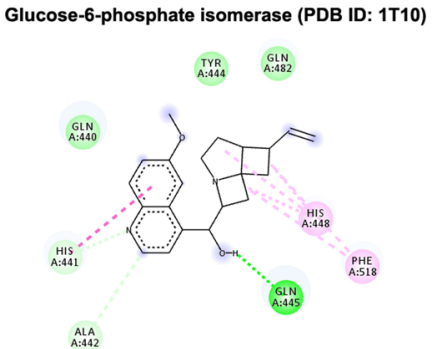

Glyceraldehyde-3-phosphate dehydrogenase (PDB ID: 1133)

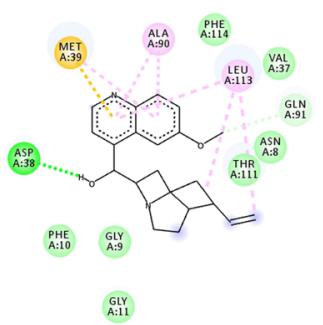

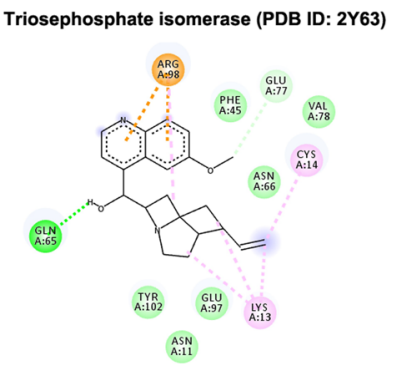

Pyruvate kinase (PDB ID: 3PP7)

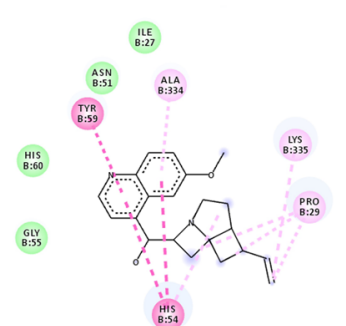

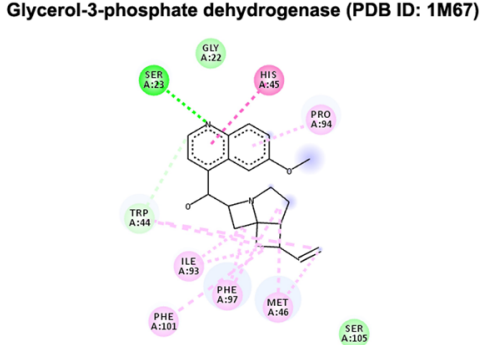

Interactions

$\square$ van der Waals

Conventional Hydrogen Bond

$\square$ Carbon Hydrogen Bond

Pi-Pi Stacked

Pi-Pi T-shaped

- Pi-Cation

$\square$ Pi-Sulfur

$\square$ Alkyl

$\square$ Pi-Alkyl

Figure 4. Quinine Interaction with Different Amino Acid Residues of Glycolytic Enzymes

Glucose-6-phosphate isomerase (PDB ID: 1T10), Triosephosphate isomerase (PDB ID: 2Y63), Glycerol-3-phosphate dehydrogenase (PDB ID: 1M67), Glyceraldehyde-3-phosphate dehydrogenase (PDB ID: 1I33), pyruvate kinase (PDB ID: 3PP7). Different kinds of interaction between amino acid residues and quinine were demonstrated inside the figure. Interactions were visualized by BIOVIA DVS.

key residues of flexible loops (residues 168178) and (residues 210-213) (Figure 3, 4 and 5) 18. Pentamidine reported relatively weak binding affinity $(-5.41 \mathrm{Kcal} / \mathrm{mol})$ compared to the anti-malarial compounds (-7.78, -6.12 , and $-6.11 \mathrm{Kcal} / \mathrm{mol}$ for artesunate, quinine and mefloquine, respectively). As for glycerol-3-phosphate dehydrogenase (GPDH), the docked pentamidine was confined to the residues that make the hydrophobic cleft, where its benzene rings formed a $\pi$-sulphur interaction with MET46 , and other interactions were observed with the residues of the hydrophobic pocket with a good binding affinity of $-7.69 \mathrm{Kcal} /$ mol. While the compounds have shown different biochemical interactions within the hydrophobic cleft and pocket composed of the side chains of MET-46, PHE-97, TRP-44, ILE-93, and PHE-101 residues.
As for the enzyme glyceraldehyde-3phosphate dehydrogenase(GAPDH), docking results for pentamidine showed nearly similar pattern of interactions to that of the antimalarial compounds. It has formed hydrogen bonding with the catalytic residue GLN-91 and $\pi$-alkyl interaction with MET-39 located in the hydrophobic cleft. The binding energy for pentamidine was reported $-6.85 \mathrm{Kcal} / \mathrm{mol}$. The docking results for the other compounds revealed biochemical interactions with residues of the fairly hydrophobic region including MET-39, LEU-113, PHE-114 and the side chain methylene units of ARG-92.

Artesunate and mefloquine formed interesting hydrogen and halogen bonding interactions with GLN-91, respectively, as this residue was required to be conserved in the design of the inhibitor N6 substituents. ${ }^{16}$ Mefloquine 

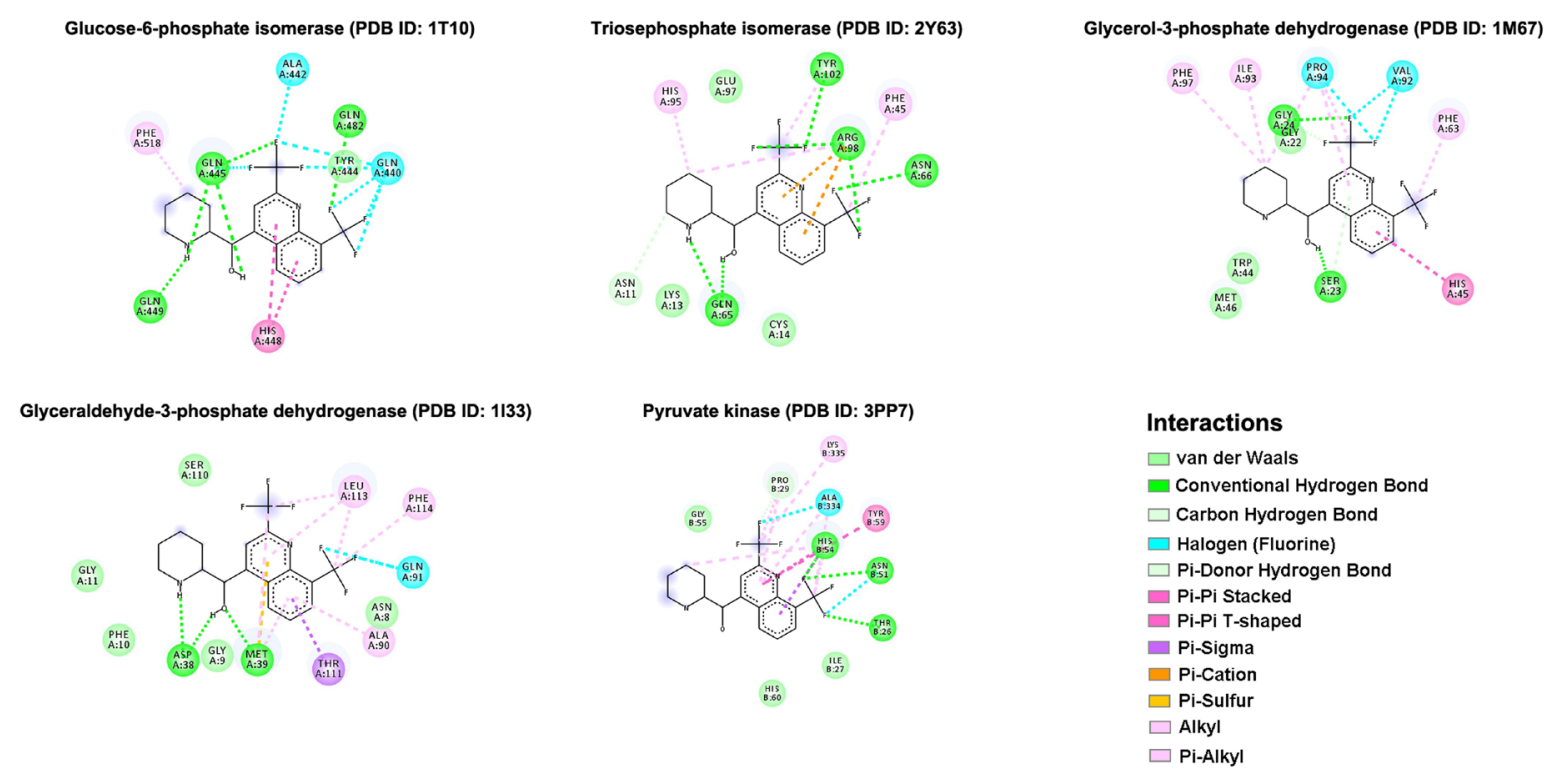

Figure 5. Mefloquine Interaction with Different Amino Acid Residues of Glycolytic Enzymes

Glucose-6-phosphate isomerase (PDB ID: 1T10), Triosephosphate isomerase (PDB ID: 2Y63), Glycerol-3-phosphate dehydrogenase (PDB ID: 1M67), Glyceraldehyde3-phosphate dehydrogenase (PDB ID: 1I33), pyruvate kinase (PDB ID: 3PP7). Different kinds of interaction between amino acid residues and mefloquine were demonstrated inside the figure. Interactions were visualized by BIOVIA DVS.

and quinine showed hydrogen bonding and $\pi$-sulphur interactions with MET-39 located in the hydrophobic cleft; a region exploit for the design of potent selective inhibitors. ${ }^{16}$ Moreover, they have shown hydrogen bonding with the conserved residue ASP38. The hydrophobic substituents that pack against LEU-113 gain affinity as well as selectivity. ${ }^{16}$ Herein, the docked compounds showed $\pi$-alkyl interactions with LEU-113.

Finally, the enzyme pyruvate kinase (PYK) catalyzes the last step reaction of glycolysis in which P-enolpyruvate and ADP are converted into pyruvate and ATP. ${ }^{32}$ Pentamidine showed $\pi-\pi$ stack and hydrogen interactions with the hydrophobic residues PRO-29 and TYR-59, respectively. Other conventional hydrogen bondings were observed with the residues HIS-54, ASN-51, THR-26, and ILE-27. The binding energy for pentamidine was reported $-6.22 \mathrm{Kcal} / \mathrm{mol}$. Similarly, docking results of the compounds showed stacking interactions with vital residues in the active pocket as TYR-59, HIS-54 and PRO-29 ${ }^{13}$. The two hydrophobic residues PRO-29 and TYR-59 are essential and provide stability via helping to hold the molecule in the ATP/ADP binding site of the enzyme PKY. ${ }^{13}$ Artesunate and mefloquine formed conventional hydrogen bonding with some of these residues, while quinine has formed van der Waals and $\pi-\pi$ stacked interactions.

Table 1 indicates that artesunate and quinine showed the lowest binding energies (-9.31 and $-7.43 \mathrm{kcal} / \mathrm{mol}$, respectively) when docked with glycerol-3-phosphate dehydrogenase (PDB ID: 1M67). While mefloquine showed lowest binding energy $(-6.91 \mathrm{kcal} / \mathrm{mol})$ when 

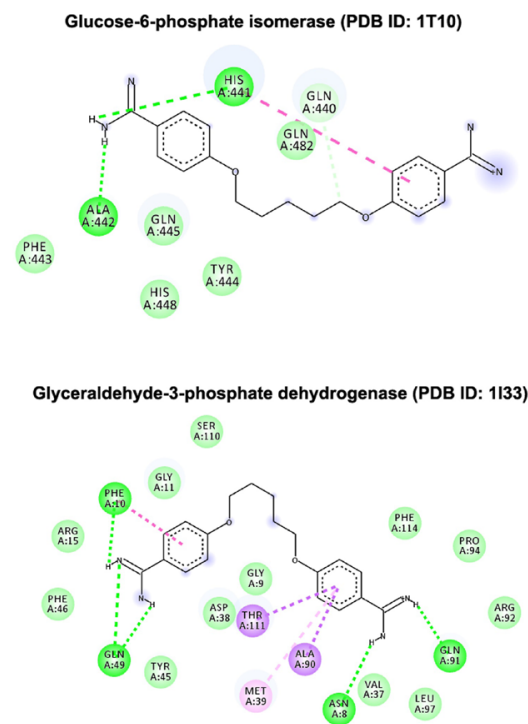
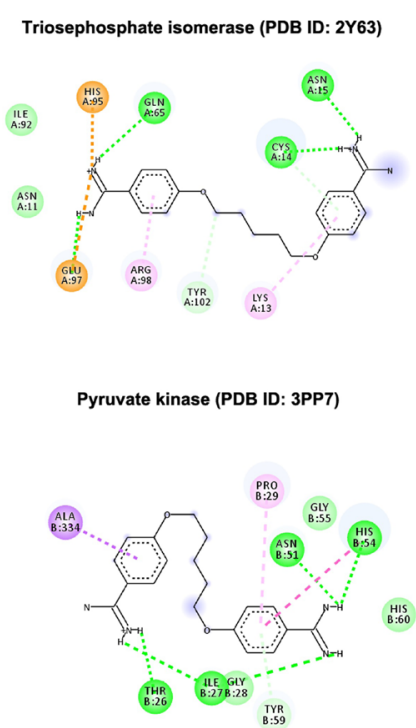
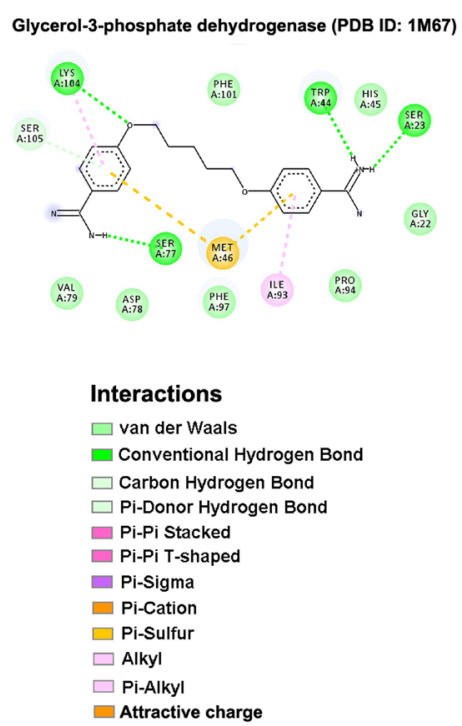

Figure 6. Pentamidine Interaction with Different Amino Acid Residues of Glycolytic Enzymes;

Glucose-6-phosphate isomerase (PDB ID: 1T10), Triosephosphate isomerase (PDB ID: 2Y63), Glycerol-3-phosphate dehydrogenase (PDB ID: 1M67), Glyceraldehyde3-phosphate dehydrogenase (PDB ID: 1I33), pyruvate kinase (PDB ID: 3PP7). Different kinds of interaction between amino acid residues and mefloquine were demonstrated inside the figure. Interactions were visualized by BIOVIA DVS

docked with glyceraldehyde-3-phosphate dehydrogenase (PDB ID: 1I33).

\section{Conclusions}

The preliminary results of this work showed relatively promising activities of artesunate, quinine and mefloquine against leishmania donovani promastigote. The in silico analysis suggested that anti-leishmanial activities mediated through inhibition of glycolysis prolqacess in the parasite. Artesunate and quinine showed better interactions, in terms of binding energies, when docked with glycerol-3-phosphate dehydrogenase, while mefloquine showed lowest binding energy when docked with glyceraldehyde3-phosphate dehydrogenase. Further future studies should focus on their detailed mechanism of action on in vivo models for the development of potential therapeutic agents.

\section{Acknowledgements}

Authors would like to acknowledge all members of the Department of Microbiology, Medicinal, Aromatic Plants and Traditional Medicine Research Institute for helping us to conduct the in vivo antileishmanial activity experiment.

\section{Funding \\ None}

\section{Conflict of Interest}

The authors have declared no conflict of interests. 


\section{References}

1. Torres-Guerrero E, Quintanilla-Cedillo MR, Ruiz-Esmenjaud J, Arenas R. Leishmaniasis: a review. F1000 Research. 2017;6:750.

2. Steverding D. The history of leishmaniasis. Parasites \& Vectors 2017;10(1):82.

3. World Health Organization. Leishmaniasis. World Health Org Fact Sheet. 2016;375. http://www.who. int/mediacentre/factsheets/fs 375/en/. Accessed 23 April 2020.

4. Alvar J, Vélez ID, Bern C, Herrero M, Desjeux P, Cano J, et al. Leishmaniasis worldwide and global estimates of its incidence. PloS One. 2012;7(5):e35671.

5. Zijlstra E, El-Hassan A. Leishmaniasis in Sudan. 3. Visceral leishmaniasis. Transactions of the Royal Society of Tropical Medicine and Hygiene. 2001;95(Suppl 1):S27-S58.

6. Siddig $M$, Ghalib $H$, Shillington D, Petersen E, Khidir S. Visceral leishmaniasis in Sudan. Clinical Features. Tropical and Geographical Medicine. 1990;42(2):107-12.

7. Al-Salem W, Herricks JR, Hotez PJ. A review of visceral leishmaniasis during the conflict in South Sudan and the consequences for East African countries. Parasites \& Vectors. 2016;9(1):460.

8. Seaman J, Mercer AJ, Sondorp HE, Herwaldt BL. Epidemic visceral leishmaniasis in southern Sudan: treatment of severely debilitated patients under wartime conditions and with limited resources. Annals of Internal Medicine. 1996;124(7):664-72.

9. Subramanian A, Sarkar RR. Revealing the mystery of metabolic adaptations using a genome scale model of Leishmania infantum. Scientific Reports. 2017;7(1):112.

10. Chawla B, Madhubala R. Drug targets in Leishmania. Journal of Parasitic Diseases. 2010;34(1):1-13.

11. Opperdoes FR, Michels PA. The metabolic repertoire of Leishmania and implications for drug discovery. In Leishmania After the Genome. P.J. Myler, and N. Fasel (eds). Caister Academic Press, pp. 123- 158.

12. Raj S, Sasidharan S, Balaji S, Saudagar P. An overview of biochemically characterized drug targets in metabolic pathways of Leishmania parasite. Parasitology Research. 2020;119:20252037.

13. Morgan HP, McNae IW, Nowicki MW, Zhong W, Michels PA, Auld DS, et al. The trypanocidal drug suramin and other trypan blue mimetics are inhibitors of pyruvate kinases and bind to the adenosine site. Journal of Biological Chemistry. 2011;286(36):31232-40.

14. Cordeiro AT, Michels PA, Delboni LF, Thiemann $\mathrm{OH}$. The crystal structure of glucose-6-phosphate isomerase from Leishmania mexicana reveals novel active site features. European Journal of Biochemistry. 2004;271(13):2765-72.

15. Führing J, Cramer JT, Routier FoH, Lamerz A-C, Baruch P, Gerardy-Schahn $\mathrm{R}$, et al. Catalytic mechanism and allosteric regulation of UDP-glucose pyrophosphorylase from Leishmania major. ACS Catalysis. 2013;3(12):297685.

16. Suresh S, Bressi JC, Kennedy KJ, Verlinde CL, Gelb MH, Hol WG. Conformational changes in Leishmania mexicana glyceraldehyde-3-phosphate dehydrogenase induced by designed inhibitors. Journal of Molecular Biology. 2001;309(2):423-35.

17. Choe J, Suresh S, Wisedchaisri G, Kennedy KJ, Gelb MH, Hol WG. Anomalous differences of light elements in determining precise binding modes 
of ligands to glycerol-3-phosphate dehydrogenase. Chemistry \& Biology. 2002;9(11):1189-97.

18. Venkatesan R, Alahuhta M, Pihko PM, Wierenga RK. High resolution crystal structures of triosephosphate isomerase complexed with its suicide inhibitors: The conformational flexibility of the catalytic glutamate in its closed, liganded active site. Protein Science. 2011;20(8):138797.

19. Fyfe PK, Westrop GD, Silva AM, Coombs GH, Hunter WN. Leishmania TDR1 structure, a unique trimeric glutathione transferase capable of deglutathionylation and antimonial prodrug activation. Proceedings of the National Academy of Sciences. 2012;109(29):11693-8.

20. Kedzierski L, Malby RL, Smith BJ, Perugini MA, Hodder AN, Ilg $\mathrm{T}$, et al. Structure of Leishmania mexicana phosphomannomutase highlights similarities with human isoforms. Journal of Molecular Biology. 2006;363(1):21527.

21. Croft SL, Seifert K, Yardley V. Current scenario of drug development for leishmaniasis. The Indian Journal of Medical Research. 2006;123(3):399-410.

22. Croft SL, Sundar S, Fairlamb AH. Drug resistance in leishmaniasis. Clinical Microbiology Reviews. 2006;19(1):11126.

23. van den Bogaart E, Berkhout MMZ, Nour ABYM, Mens PF, Talha A-BA, Adams ER, et al. Concomitant malaria among visceral leishmaniasis in-patients from Gedarif and Sennar States, Sudan: a retrospective case-control study. $B M C$ Public Health. 2013;13:332

24. Gomez Lea, Andrial M, Hosokawa A, Nonaka S, Hahiguchi Y. Oral Treatment of New World Cutaneous Leishmaniasis with Anti-Malarial Drugs in ECUADOR: A Preliminary Clinical
Trial. 1995;23(3):151-7.

25. Mwololo SW, Mutiso JM, Macharia JC, Bourdichon AJ, Gicheru MM. In vitro activity and in vivo efficacy of a combination therapy of diminazene and chloroquine against murine visceral leishmaniasis. International Journal of Biomedical Research. 2015;29(3):21423.

26. Tian W, Chen C, Lei X, Zhao J, Liang J. CASTp 3.0: computed atlas of surface topography of proteins. Nucleic Acids Research. 2018;46(W1):W363-W7.

27. Morris GM, Huey R, Lindstrom W, Sanner MF, Belew RK, Goodsell DS, et al. AutoDock4 and AutoDockTools4: Automated docking with selectivereceptor flexibility. Journal of Computational Chemistry. 2009;30(16):2785-91.

28. Morris GM, Goodsell DS, Halliday RS, Huey R, Hart WE, Belew RK, et al. Automated docking using a Lamarckian genetic algorithm and an empirical binding free energy function. Journal of Computational Chemistry. 1998;19(14):1639-62.

29. Biovia DS. Discovery Studio Modeling Environment; Dassault Systèmes: San Diego, CA, USA, 2015.

30. Nettey $H$, allotey-babington L, N'Guessan B, Afrane B, Tagoe M, Ababio A, et al. Screening of Anti-Infectives against Leishmania donovani. Advances in Microbiology. 2016;6(1):13-22.

31. Allotey-Babington GL, Amponsah SK, Nettey T, Sasu C, Nettey H. Quinine Sulphate Microparticles as Treatment for Leishmaniasis. Journal of Tropical Medicine. 2020;30:5278518.

32. Pinzi L, Rastelli G. Molecular Docking: Shifting Paradigms in Drug Discovery. International Journal of Molecular Sciences. 2019;20(18):4331.

33. Rocha V, Nonato F, Guimaraes E, Freitas L, Soares M. Activity of antimalarial 
drugs in vitro and in a murine model of cutaneous leishmaniasis. Journal of Medical Microbiology. 2013;62(70): 1001-10.

34. Yang DM, Liew FY. Effects of qinghaosu (artemisinin) and its derivatives on experimental cutaneous leishmaniasis. Parasitology. 1993;106(1):7-11.

35. Smith DF, Peacock CS, Cruz AK. Comparative genomics: from genotype to disease phenotype in the leishmaniases. International Journal of Parasitology. 2007;37(11):1173-86.

36. Opperdoes FR, Borst P. Localization of nine glycolytic enzymes in a microbodylike organelle in Trypanosoma brucei: the glycosome. FEBS Letters. 1977;80(2):360-4.

37. Michels PAM, Bringaud F, Herman M, Hannaert V. Metabolic functions of glycosomes in trypanosomatids. Biochimica et BiophysicaActa-Molecular Cell Research. 2006;1763(12):1463-77. 